\title{
A neck-line structure in the dust tail of Comet C/2004 F4 (Bradfield)
}

\author{
L. Pansecchi and M. Scardia
}

\author{
INAF - Osservatorio Astronomico di Brera, via E. Bianchi 46, 23807 Merate LC, Italy \\ e-mail: luigi.pansecchi@libero.it; scardia@merate.mi.astro.it
}

Received 1 August 2004 / Accepted 4 September 2004

\begin{abstract}
Three CCD images of Comet C/2004 F4 (Bradfield), taken on three different dates close to the time of the Earth crossing through the plane of the comet orbit (2004 May 2.9280 UT), show a ray-shaped structure (RSS) in the dust tail, accompanied by a short, sunward spike (SWS). This morphology and behaviour strongly recall a Neck-Line Structure (NLS; Kimura \& Liu 1977; Pansecchi et al. 1987). Astrometric measurements of the images show that, on all the three dates, the position on the sky of the observed RSS is consistent with several sky-projected points of a theoretical Neck-Line, computed for the precise date of each observation. Furthermore, the SWS turns out to be a real (not caused by perspective) antitail, which is predicted by NLS models.
\end{abstract}

Key words. comets: general - comets: individual: C/2004 F4 (Bradfield)

\section{Introduction}

A Neck-Line Structure (NLS) is a special dusty feature that was found by Kimura \& Liu (1977) in the development of their theoretical model of cometary dust tails. In such a model, each dust particle is taken as an infinitely small planetary body, whose orbital elements, and then its position in space at any time, can be rigorously computed using Keplerian orbit mechanics. Consequently, because of the Keplerian motion of the individual particles, the initial spherical (or quasi-spherical, depending on the ejection geometry) shells, ejected from the nucleus before perihelion, remain spherical for a while after emission. Soon they become ellipsoidal in shape by collapsing after perihelion onto the orbital plane of the parent comet at the second node of the particle orbits, that is, at the point $\pi$ away in true anomaly from the ejection point (the first node). At the second node, the initial three-dimensional shells are completely flattened on the plane of the comet orbit. The ideal line connecting the comet nucleus with all the second nodes of the particle orbits (in practice with the centers of the flattened ellipsoids) is called the Neck-Line (NL), and the resulting flat structure of dust particles of different sizes and different emission times that cross the comet orbital plane (each in its second node) at a given instant of time is called the NLS for that instant. As shown by Pansecchi et al. (1987), such a structure has a short, sunward extension. In their NLS model, the dust ellipsoids composed of the largest dust grains, on which the radiation pressure force has negligible effects, after having collapsed onto the plane of the comet orbit, form in such a plane a flat ellipse. This ellipse is approximately centered on the comet nucleus, and its major axis is nearly parallel to the sunward prolongation of the NL. Therefore half of such an ellipse lies in the inner part of the comet orbit, thus resulting in a real antitail (see Fig. 4 in the paper by Pansecchi et al. 1987). In general, two conditions should be satisfied for a NLS to be observed in a well developed dust tail: (i) the comet must have passed its perihelion point; (ii) the Earth must be close to the plane of the comet orbit. The first condition is imposed by the spatial location of the second node of the particle orbits. For the second one, the resulting edgewise (or quasi-edgewise) perspective allows better visibility of the NLS, because of the piling up of the dust particles along the line of sight. Under such conditions, a NLS is expected to look like a ray-shaped structure (RSS), stretching from the nucleus across a regular dust tail, extending somewhat sunward in a short, narrow antitail (i.e., in a sunward spike; SWS). It is surrounded by a fainter envelope representing the edgewise projection on the sky of the three-dimensional part of the tail, that is, of the collapsing ellipsoids composed of dust ejected continuously by the comet nucleus. NLSs were identified in the dust tails of at least seven comets: Arend-Roland 1957III (Kimura \& Liu 1977), Bennett 1970II (Pansecchi et al. 1987), Halley 1986III (Cremonese \& Fulle 1989), Great January Comet 1910I (Pansecchi \& Fulle 1990), Austin 1990V (Fulle et al. 1993), Hale-Bopp 1995 O1 (observed at the beginning of 1998; Fulle 2004), and 67P/Churyumov-Gerasimenko (Fulle et al. 2004).

In this paper we present and analyse three photographs of Comet C/2004 F4 (Bradfield), taken after perihelion, around 


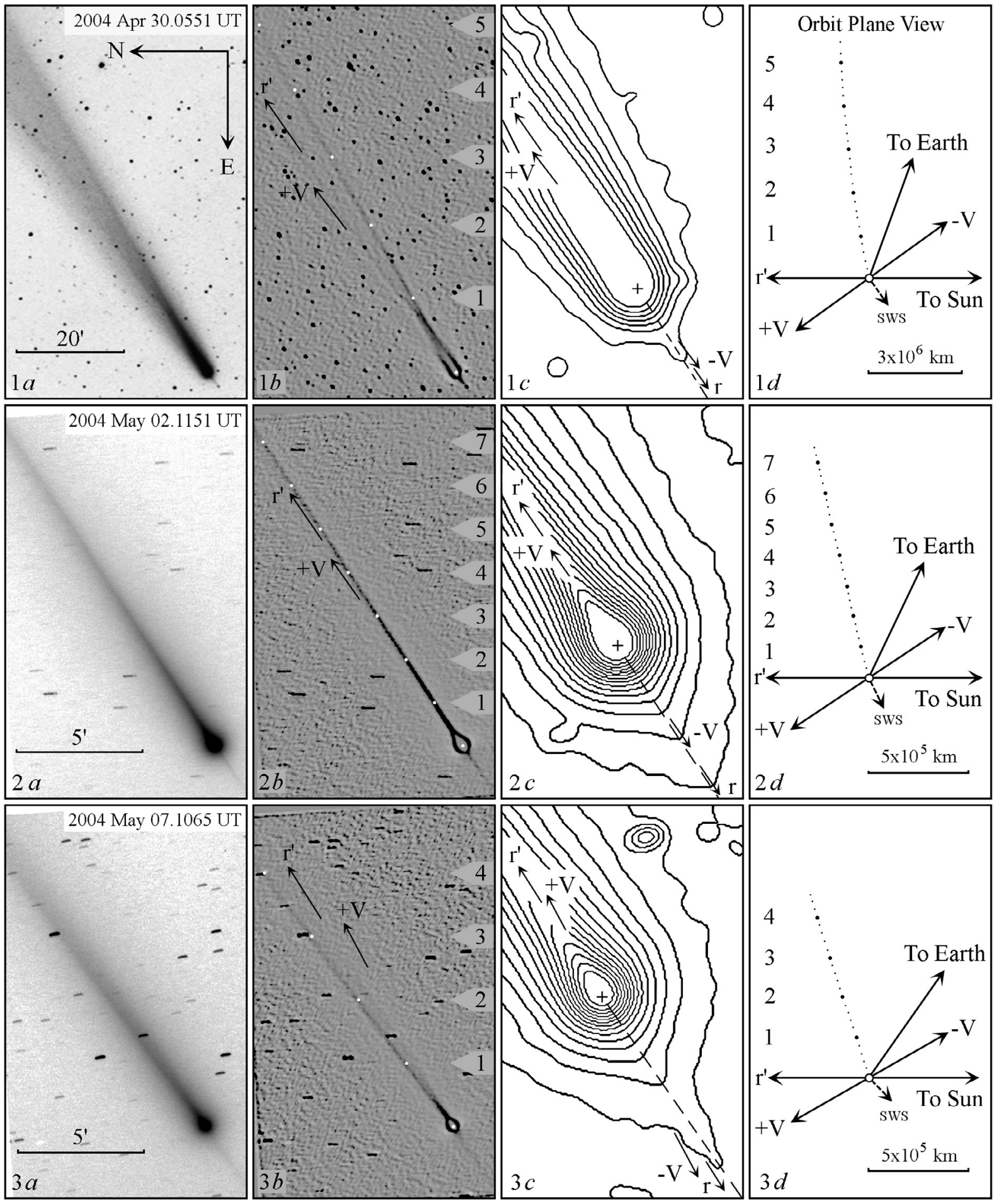

Fig. 1. Observations and related reductions: in $\boldsymbol{a}$ ), the original CCD images; in $\boldsymbol{b}$ ), a processed version of them; in $\boldsymbol{c}$ ), isophotes of the near-coma area; in $\boldsymbol{d}$ ), orbit plane views with the projected orientation of the sunward spike (dashed arrows), and the computed points of the theoretical Neck Line (black dots), sequentially numbered from the nucleus. Such points are projected on the plane of the sky, into the processed images in $\boldsymbol{b}$ ), as white dots with the same sequential number. Arrows $\mathrm{r}, \mathrm{r}^{\prime},+\mathrm{V}$, and $-\mathrm{V}$, denote the direction to the Sun from the nucleus, the prolonged Sun-comet radius vector, and the tangential orbital velocity vector of the nucleus in the sense of the comet motion, and in the opposite sense. Arrows $\mathrm{N}$ and $\mathrm{E}$ denote North and East through the nucleus.

the time of the Earth crossing through the plane of the comet orbit, and therefore under the ideal conditions described above. All the photographs show a RSS in the dust tail, accompanied by a short SWS, which, on the whole, strongly resemble a NLS.
Our analysis will consist in comparing the position on the sky of the observed structures with some sky-projected points of a theoretical NL, by means of astrometric measurements of the images. 
Table 1. Observational data and geometrical circumstances. No: Serial number of the observation. Obs: Observer (DT - Diego Tirelli, Italian Group of Comet Observers; MJ \& GR - Michael Jäger \& Gerald Rhemann, Astrostudio G. Rhemann Ges. m. b. h). Date UT (2004): Time at midexposure. RA, Dec: right ascension and declination of the comet nucleus, respectively, measured directly on the CCD images through astrometric calibration. $\Delta, r$ : Earth-comet and Sun-comet distances. $\phi$ : Phase angle. $v$ : True anomaly of the comet nucleus. $\beta$ : Cometocentric latitude of the Earth; it is positive when the Earth is in the comet north hemisphere.

\begin{tabular}{cccccccccr}
\hline \hline No & Obs. & Date UT (2004) & RA $\left[^{\circ}\right]$ & Dec $\left[{ }^{\circ}\right]$ & $\Delta[\mathrm{AU}]$ & $r[\mathrm{AU}]$ & $\phi\left[^{\circ}\right]$ & $v\left[^{\circ}\right]$ & $\beta\left[^{\circ}\right]$ \\
\hline 1 & MJ \& GR & Apr. 30.0551 & 14.6486 & 32.5708 & 1.0628 & 0.4930 & 70.0 & 108.533 & 2.4 \\
2 & DT & May 02.1151 & 14.6211 & 34.6623 & 1.1141 & 0.5500 & 64.5 & 112.873 & 0.6 \\
3 & DT & May 07.1065 & 15.1268 & 38.7339 & 1.2317 & 0.6818 & 55.0 & 120.463 & -3.0 \\
\hline
\end{tabular}

\section{Observations and reduction procedure}

According to the orbital elements we used in this paper (Marsden 2004), Comet C/2004 F4 passed its perihelion point on 2004 April 17.0899 UT. Nearly sixteen days later, on May 2.9280 UT, the Earth crossed the plane of the comet orbit. The observations analysed here consist of three CCD images, taken on three different dates, at $-2.9,-0.8$, and +4.2 days from the date of the Earth crossing. The original photographs, labelled $a$, are shown in the left-hand column of Fig. 1. The quantities describing the relevant geometrical circumstances and projection conditions are summarized in Table 1 . The first observation (Fig. 1.1a) is a wide-field, unfiltered photograph, taken with a $20 / 30 \mathrm{~cm}$ Schmidt camera, whereas the others (Figs. 1.2a, and 1.3a) are images of much smaller field, taken with a $25-\mathrm{cm}, \mathrm{f} / 6$ reflector, through a $R$ and I-Cousins filter, centered at $647.2 \mathrm{~nm}$ and $786.9 \mathrm{~nm}$, with a total WHM of $261.3 \mathrm{~nm}$ (Moro \& Munari 2000). The original images have been astrometrically calibrated by means of the field stars and then processed in order to isolate and emphasize the axis (i.e., the line of maximum density or maximum brightness) of the RSS for a comparison with a theoretical NL. These processed images are assembled in Col. $b$ of Fig. 1. A series of isophotes of the area near the coma were also obtained to measure the orientation of the SWS (Col. $c$ of Fig. 1). Here the dashed line connecting the position of the comet nucleus (marked with a small cross) with the vertices of the isophotes was taken as denoting the position angle on the sky of the SWS (PA $\mathrm{PAS}_{\mathrm{SW}}$ in Table 2). We also tried to measure the length of the SWS, but, after some attempts, we abandoned this idea, the results being too uncertain because of three factors: the general faintness of such a feature; the small scale and star pollution in observation 1; the fact that, in observations 2 and 3, the SWS seems to extend outside the field. The measured $\mathrm{PA}_{\mathrm{SWS}}$ was then projected on the plane of the comet orbit, into the diagrams in Col. $d$, where the orientation of the SWS is represented by a short, dashed arrow. All the photographs (and the derived images) in Fig. 1 have the same orientation, and arrows $\mathrm{N}$ and $\mathrm{E}$ in Fig. 1.1 $a$ indicate North and East through the nucleus. For the precise date of each observation, we then computed several points along the theoretical NL in the plane of the comet orbit, following the procedure described by Pansecchi at al. (1987, 1988). The diagrams with the orbit plane views are shown in the last column of Fig. 1. The black dots, sequentially numbered from the nucleus, are the computed centers of the dust shells, collapsed (flattened) onto the orbit plane of the parent comet at the second node of the particle orbits. Each point is characterized by zero ejection velocity from the comet nucleus and by a given value of $(1-\mu)$, the solar radiation pressure force acting on the dust grain expressed in solar gravitational force units, and of $\tau$, the time (in days) between dust ejection and observation. All the parameters concerning the computed NL points are summarized in the right hand part of Table 2. The ideal (dotted) line connecting the black dots with the comet nucleus (unfilled dot) is the NL for the instant of time of the observation. Finally, the computed NL points have been projected on the plane of the sky, into the processed images in Col. $b$ of Fig. 1, as white dots with the same sequence number. Figure 1 clearly shows that, on the three dates, the sky-projected points of the theoretical NL closely fit the axis of the observed RSS. In Table 2, the average position angle of the RSS ( $\mathrm{PA}_{\mathrm{RSS}}$ ), measured directly on the photographs, is also given. The precise orientation on the sky of the RSS, at various distances from the nucleus, is represented by the position angles of the single, sky-projected, NL points $\left(\mathrm{PA}_{\mathrm{NL}}\right.$ in Table 2).

\section{Discussion and conclusion}

The results of the astrometric measurements described in the previous section show that, throughout the relevant time, the position on the sky of the RSS is consistent with several skyprojected points of a theoretical Neck-Line. We could conclude that the RSS in the dust tail of Comet C/2004 F4 (Bradfield) is really a NLS. However, two objections might be raised: (i) the RSS might be a plasma feature; or (ii) a layer of dust (DL) widespread on the plane of the comet orbit, that we see as a RSS only because of the particular projection conditions. The filter used in observations 2 and 3 appears to be insufficient to suppress all plasma features. Indeed, a faint trace of the plasma tail is still visible in the image of May 7 (Figs. 1.3a, and 1.3b), south of the RSS, up to a distance from the nucleus of $\sim 8^{\prime}$. But, fortunately, such an image also shows that the plasma tail lies nearly along the prolonged Sun-comet radius vector $\left(r^{\prime}\right)$, in a position angle quite different from that of the RSS. Therefore, the two structures cannot be confused. The same thing can be said for the unfiltered image of Apr. 30 (Figs. 1.1 $a$ and 1.1b), where the two structures appear to be clearly separated. On the other hand, the fact that the sky-projected NL points coincide with the RSS implies, of course, that, if reduced to the plane of the comet orbit, such a structure would coincide with the NL points in that plane. Now, it is easy to observe from the orbit plane views in Fig. 1 or from the orbital position angles $\left(\mathrm{oPA}_{\mathrm{NL}}\right)$ in Table 2 that the aberration angle of such points 
Table 2. RSS, SWS and NL parameters. No.: serial number of the observation. $\mathrm{PA}_{\mathrm{RSS}}$ : measured position angle on the sky of the average orientation of the RSS. PA $\mathrm{SWS}_{\mathrm{S}}$ : measured position angle on the sky of the SWS. PA $\mathrm{RV}_{\mathrm{RV}}$ : computed position angle on the sky of the Sun-comet radius vector. $\mathrm{PA}_{+\mathrm{V}}$ : computed position angle on the sky of the positive velocity vector. NLp: NL point sequential number $($ Fig. 1$)$. $(1-\mu)$ : radiation pressure acting on the dust grain. $\tau$ : days elapsed between ejection of the dust grain from the comet nucleus and observation. $d\left[^{\circ}\right]$, $d\left[10^{6} \mathrm{~km}\right]$ : angular (in the plane of the sky) and linear (in the plane of the comet orbit) distance from the nucleus of the computed NL point, respectively. $\mathrm{PA}_{\mathrm{NL}}$ : position angle of the sky-projected $\mathrm{NL}$ point. oPA $\mathrm{NL}_{\mathrm{NL}}$ : orbital position angle of the $\mathrm{NL}$ point. Position angles in the plane of the sky are measured from north to east, as usual, whereas the ones in the plane of the comet orbit (prefix o) are measured counterclockwise from the Sun-comet prolonged radius vector $\left(\mathrm{r}^{\prime}\right)$.

\begin{tabular}{|c|c|c|c|c|c|c|c|c|c|c|c|}
\hline No. & $\mathrm{PA}_{\mathrm{RSS}}\left[^{\circ}\right]$ & $\mathrm{PA}_{\mathrm{SWS}}\left[{ }^{\circ}\right]$ & $\mathrm{PA}_{\mathrm{RV}}\left[^{\circ}\right]$ & $\mathrm{PA}_{+\mathrm{V}}\left[{ }^{\circ}\right]$ & NLp & $(1-\mu)$ & $\tau$ [days] & $d\left[^{\circ}\right]$ & $\mathrm{PA}_{\mathrm{NL}}\left[^{\circ}\right]$ & $d\left[10^{6} \mathrm{~km}\right]$ & $\mathrm{oPA}_{\mathrm{NL}}\left[{ }^{\circ}\right]$ \\
\hline \multirow[t]{5}{*}{1} & 299.9 & 122.5 & 125.01 & 307.61 & 1 & 0.01 & 17.87 & 0.259 & 300.23 & 1.385 & 280.97 \\
\hline & & & & & 2 & 0.02 & 17.99 & 0.510 & 300.09 & 2.775 & 280.11 \\
\hline & & & & & 3 & 0.03 & 18.12 & 0.753 & 299.94 & 4.172 & 279.24 \\
\hline & & & & & 4 & 0.04 & 18.26 & 0.986 & 299.79 & 5.573 & 278.36 \\
\hline & & & & & 5 & 0.05 & 18.39 & 1.210 & 299.62 & 6.981 & 277.47 \\
\hline \multirow[t]{7}{*}{2} & 303.3 & 123.6 & 124.41 & 305.17 & 1 & 0.001 & 19.35 & 0.035 & 303.33 & 0.158 & 283.84 \\
\hline & & & & & 2 & 0.002 & 19.36 & 0.069 & 303.33 & 0.316 & 283.76 \\
\hline & & & & & 3 & 0.003 & 19.37 & 0.103 & 303.32 & 0.475 & 283.67 \\
\hline & & & & & 4 & 0.004 & 19.38 & 0.138 & 303.32 & 0.633 & 283.58 \\
\hline & & & & & 5 & 0.005 & 19.39 & 0.172 & 303.32 & 0.791 & 283.50 \\
\hline & & & & & 6 & 0.006 & 19.40 & 0.206 & 303.32 & 0.949 & 283.41 \\
\hline & & & & & 7 & 0.007 & 19.41 & 0.240 & 303.31 & 1.108 & 283.32 \\
\hline \multirow[t]{4}{*}{3} & 306.7 & 125.0 & 122.37 & 298.17 & 1 & 0.001 & 23.62 & 0.052 & 306.68 & 0.210 & 287.86 \\
\hline & & & & & 2 & 0.002 & 23.63 & 0.104 & 306.68 & 0.420 & 287.77 \\
\hline & & & & & 3 & 0.003 & 23.64 & 0.156 & 306.69 & 0.630 & 287.68 \\
\hline & & & & & 4 & 0.004 & 23.65 & 0.208 & 306.70 & 0.840 & 287.60 \\
\hline
\end{tabular}

(difference with respect to the antisolar direction $=2 \pi-\mathrm{oPA}_{\mathrm{NL}}$ ) would be too large (between $72^{\circ}$ and $82^{\circ}$, depending on the date and on the distance from the nucleus) to be consistent with a plasma tail, unless the RSS is outside the plane of the comet orbit. Furthermore, the general shape of the RSS does not change substantially over the relevant time (seven days), and its PARSS clearly changes only with the changing projection conditions. Both such behaviours are characteristic of a dusty feature. As to objection (ii), we acknowledge that for the image of May 2, taken when the Earth was almost exactly in the plane of the comet's orbit (see parameter $\beta$ in Table 1), it is impossible to discriminate between the NLS model and the DL model, because of the too severe projection conditions. The situation is however more favourable for the observations of April 30 and May 7. We consider it unlikely (although not impossible) that the consistency of the sky-projected NL points with the RSS on the three dates may be a coincidence. But, as already pointed out by Pansecchi et al. (1987), the discriminating criterion may be the presence of the SWS. Astrometric measurements show that such a feature lies on the sky nearly along the sunward prolongation of the RSS, in the sector delimited by the Suncomet radius vector $(\mathrm{r})$ and by the comet velocity vector $+\mathrm{V}$ (see $\mathrm{PA}_{\mathrm{RV}}$ and $\mathrm{PA}_{+\mathrm{V}}$ in Table 2). Figure $1.3 c$ offers the best evidence of such a geometry. On the (reasonable) assumption that such a structure is confined to the plane of the comet orbit, this means that the SWS lies inside the path of the comet orbit with respect to the Sun, and therefore should be considered as a real (not-perspective) antitail in the Bredikhin sense (Jaegermann 1903). As explained in Sect. 1, such a real antitail is predicted by the NLS model of Pansecchi et al. (1987) as a natural, sunward extension of the NLS. The orbit plane diagrams in Fig. 1 show that the projected orientation of the SWS is not parallel to the NL, as required by the model, but more or less tilted toward the Sun. This, however, might be explained by the measurement uncertainties. In conclusion, the presence of the SWS as a real antitail should be a strong argument in favour of the NLS interpretation of the RSS.

Acknowledgements. We are indebted to Michael Jäger \& Gerald Rhemann (Astrostudio G. Rhemann Ges. m. b. h.), and Diego Tirelli (Italian Group of Comet Observers; GOC), who kindly made available the photographs analysed here. Thanks are due also to Rolando Ligustri, and Giovanni Sostero (they too members of gOC) who, alerted by the first author, worked hard to secure images during the critical period, although unsuccessfully because of bad weather.

\section{References}

Cremonese, G., \& Fulle, M. 1989, Icarus, 80, 267

Fulle, M. 2004, personal communication

Fulle, M., Bosio, S., Cremonese, G., et al. 1993, A\&A, 272, 634

Fulle, M., Barbieri, C., Cremonese, G., et al. 2004, A\&A, 422, 357

Jaegermann, R. 1903, Prof. Dr. Th. Bredikhin's Mechanische Untersuchungen über Cometenform, St. Petersburg

Kimura, H., \& Liu, C.P. 1977, Chin. Astron., 1, 235

Marsden, B.G. 2004, MPEC 2004-K39

Moro, D., \& Munari, U. 2000, A\&A, 147, 361

Pansecchi, L., \& Fulle, M. 1990, A\&A, 239, 369

Pansecchi, L., Fulle, M., \& Sedmak, G. 1987, A\&A, 176, 358

Pansecchi, L., Fulle, M., \& Sedmak, G. 1988, A\&A, 205, 367 